\title{
CAMBIOS EN EL USO DEL TIEMPO DE LAS PAREJAS ¿Estamos en el camino hacia una mayor igualdad?
}

\section{CHANGES IN COUPLES' TIME USE Are we moving toward greater equality?}

\author{
Marc AJenjo Cosp majenjo@ced.uab.es \\ Centre d'Estudis Demogràfics. Universitat Autònoma de Barcelona. España.
}

JoAN GaRcía RomÁn jgarcia@ced.uab.es

Centre d'Estudis Demogràfics. Universitat Autònoma de Barcelona. España.

Minnesota Population Center. University of Minnesota. EEUU.

\begin{abstract}
RESUMEN
El artículo analiza cómo han evolucionado las desigualdades de género a partir de las dos encuestas sobre el uso del tiempo llevadas a cabo por el Instituto Nacional de Estadística en 2002-2003 y 2009-2010. En primer lugar se analizan los cambios en el uso del tiempo y las características de las parejas a nivel descriptivo. En esta primera parte se observa una disminución de las desigualdades así como un incremento de parejas con características asociadas a valores más igualitarios, entre las que destacan la ocupación y la mejor educación femenina. En segundo lugar se contrasta si existe una evolución temporal hacia la igualdad o se debe a los cambios en las características de las parejas. Los resultados nos llevan a predecir una disminución de las desigualdades por dos razones: por la proliferación de parejas con características más igualitarias y por una mayor igualdad en el seno de las parejas.
\end{abstract}

\section{Palabras Clave}

España; Pareja de doble ingreso; Roles de género; Uso del tiempo.

\section{Abstract}

The article analyzes the evolution of gender inequality in Spain using data from the time use surveys carried out by the Spanish Statistical Office in 2002-2003 and 2009-2010. Firstly, changes in time use and characteristics of the couples are analyzed in a descriptive way. In this part, a decrease in the gap as well as an increase within couples with more egalitarian characteristics, especially female employment and higher educational attainment, is shown. Secondly, is tested the evolution towards a more equality pattern or if changes are only due to an increase of more egalitarian couples. Results predict a decrease in the gender inequality in two senses: the raise of more egalitarian couples and a higher equality within couples.

\section{KEYWORDS}

Dual-Earner Couples; Gender Roles; Spain; Time Allocation. 


\section{INTRODUCCIÓN ${ }^{*}$}

El Instituto Nacional de Estadística ha llevado a cabo, mediante una metodología muy similar, dos ediciones de la Encuesta de Empleo del Tiempo durante la primera década del siglo XXI (2002-2003 y 2009-2010). Las encuestas se han realizado en dos momentos distintos del ciclo económico: la primera en pleno crecimiento, y la segunda en período de recesión. En el año 2003 la economía española se encontraba en uno de sus mejores momentos de la historia reciente, como lo indicaba una tasa de paro del $11,5 \%$, que siguió descendiendo hasta 2007, cuando alcanzó el 8,3\%. A partir de este momento la escalada del paro ha sido continua y muy importante, hasta alcanzar el $20,1 \%$ en 2010, momento en el que se ha realizado la segunda edición de la encuesta.

Aprovechando la disponibilidad de datos en dos momentos tan dispares, el artículo analiza el efecto que la coyuntura económica ha tenido en la distribución del tiempo, así como el efecto en las diferencias entre hombres y mujeres, ya que el paro ha afectado de manera desigual a la ocupación masculina y la femenina. Así, uno de los factores que ha incidido de manera más decisiva en una distribución más igualitaria del tiempo dentro de las parejas es la participación de la mujer en el mercado de trabajo. Este hecho ha comportado la creciente generalización de las parejas de doble ingreso, las cuales representan un primer avance en la igualdad de género entre hombre y mujer (Gershuny 2000; Dema 2005; Ajenjo y García 2011). Ahora bien, el cambio en la tendencia del mercado laboral experimentado en los últimos años debido a la crisis económica ha comportado cambios importantes en la tipología de pareja. En primer lugar ha supuesto un freno en el crecimiento de la proporción de parejas de doble ingreso. En contrapartida, se han incrementado las parejas donde solo trabaja la mujer y aquellas donde no trabaja ningún miembro, consecuencias de la incidencia desigual del paro entre hombres y mujeres y del aumento de la desocupación en el total de la población, respectivamente.

\section{TENDENCIAS GENERALES EN EL USO DEL TIEMPO DE LAS FAMILIAS}

Una de las consecuencias más importantes de la incorporación masiva de la mujer al mercado de trabajo ha sido un cambio muy importante en la distribución del tiempo de hombres y mujeres, que evoluciona hacia un reparto más simétrico (Sayer 2005). Los cambios se han dado especialmente entre las mujeres y los hombres en unión y tam-

\footnotetext{
"Este trabajo se inscribe dentro de los proyectos: El reparto del tiempo y el uso del espacio en las parejas biactivas: La situación española en el contexto europeo (Ref. CSO2009-08273 / GEOG) y Espacios de vida y usos del tiempo de las familias post divorcio (Ref. CSO2012-39157), subvencionados por el plan nacional I+D+I del Ministerio de Educación y Ciencia; además de formar parte de la tesis doctoral de Joan García Román. Se ha realizado con financiación del proyecto WORLDFAM, European Research Council (ERC-2009-StG-240978).
} 
bién entre aquellos con hijos, que eran los que presentaban más divergencias, siendo el comportamiento de solteros y solteras tradicionalmente más similar (South y Spitze 1994; Sayer 2005).

La tendencia en el uso del tiempo de las parejas muestra que, en los países occidentales, mientras que el trabajo remunerado llevado a cabo por las mujeres se ha doblado desde los años sesenta, su dedicación a trabajo no remunerado ha disminuido considerablemente (Bianchi et al. 2006; Sayer 2005; Gershuny 2000; Windebank 2001). Ahora bien, el incremento en trabajo remunerado no se ha visto compensado por un descenso equivalente en trabajo no remunerado, por lo que ha sido denominado como revolución estancada (stalled revolution), cuya principal consecuencia es la concatenación de la jornada laboral con las tareas domésticas por parte de las mujeres, el denominado segundo turno (Hochschild 1989; Gershuny, Godwin y Jones 1994; Gershuny 2000; Coltrane 2000; Bianchi et al. 2006). El descenso del tiempo dedicado a trabajo no remunerado se ha producido principalmente en aquellas tareas más rutinarias y arduas como la limpieza del hogar o la preparación de las comidas, entre otras (Bianchi et al. 2006; Ferree 1991; Becker y Moen 1999); mientras que también se observa cierta reducción del tiempo total dedicado a tareas domésticas como consecuencia de los avances tecnológicos (Robinson y Godbey 1997). Por el contrario, el tiempo dedicado al cuidado de los hijos, no solo no ha decrecido, sino que incluso se ha incrementado. En este caso particular, también se ha producido un descenso en las actividades más rutinarias; mientras que el tiempo dedicado a actividades más interactivas, como leer o jugar, ha aumentado (Bianchi, Robinson y Milkie 2006).

Por lo que respecta a los hombres, el trabajo remunerado realizado se ha mantenido en niveles similares, mientras que su participación en el trabajo doméstico se ha incrementado, aunque sin llegar a compensar el descenso observado entre las mujeres (Bianchi et al 2006; Gershuny 2000). Asimismo, se observa un incremento en las tareas de cuidado de los hijos, sobre todo en el tiempo dedicado a las actividades más interactivas (Bianchi et al. 2006; Becker y Moen 1999). En consecuencia, aunque se puede decir que el padre también lleva a cabo un doble turno, este es significativamente menor que el de la madre (Bianchi et al. 2006). En este sentido, el ritmo de crecimiento de la participación del hombre al trabajo no remunerado está siendo más tardío y lento que el de la participación de la mujer al trabajo remunerado, por lo que es habitual hablar de un retraso adaptativo (lagged adaptation) a la nueva situación creada ante la mayor participación de la mujer en el trabajo remunerado (Hochschild 1989; Gershuny, Godwin y Jones 1994).

En el área de estudio de los usos del tiempo, y por convención, el tiempo se suele agrupar en cuatro grandes bloques: trabajo remunerado, trabajo no remunerado, cuidado personal y ocio (Robinson y Godbey 1997). La suma de trabajo remunerado y no remunerado se denomina trabajo total (total work) (Becker 1985; Berk 1985) y es muy similar entre hombres y mujeres. Si consideramos que el tiempo dedicado a cuidado personal se ha mantenido constante y que difiere poco entre hombres y mujeres, el tiempo restante, el tiempo de ocio, también será muy similar. Sin embargo, aunque la cantidad 
no presenta grandes diferencias, si lo hace la 'calidad' del tiempo de este ocio, debido principalmente a que para las mujeres suele ser un tiempo que se simultanea con otras actividades (Bittman y Wajcman 2000; Bianchi et al. 2006).

En lo referente a las tendencias en un período más reciente, Aguiar et al. (2011) analizan para Estados Unidos lo sucedido en los últimos años haciendo hincapié en la redistribución del tiempo en época de recesión. Con datos del American Time Use Survey (ATUS) de 2003 a 2010 observan cómo un tercio de la reducción del tiempo dedicado a trabajo remunerado se destina a trabajo doméstico y a cuidado de los hijos, mientras que una quinta parte se dedica a actividades de ocio. Otra parte importante se destina a dormir y a ver la televisión, un $10 \%$ a educación y formación y a actividades cívicas, quedando una fracción muy pequeña para la búsqueda de trabajo.

\section{EL USO DEL TIEMPO COMO INDICADOR DE IGUALDAD EN ESPAÑA}

Es muy habitual utilizar las diferencias en el tiempo que hombres y mujeres destinan a trabajo no remunerado como un indicador de género. La división del trabajo no remunerado ha sido considerado un campo simbólico donde hombres y mujeres muestran sus roles de género, los cuales presuponen que la mujer ha de realizar una gran proporción de trabajo no remunerado para demostrar su feminidad y el hombre una reducida proporción para reforzar su masculinidad (West y Zimmerman 1987; Coltrane 2000).

En España, el estudio de las diferencias de género a partir del empleo del tiempo se remonta a los años setenta (Durán y Rogero 2009). Impulsada por la profesora María Ángeles Durán y focalizando principalmente en el reparto del trabajo no remunerado, esta nueva línea de investigación tuvo que afrontar la dificultad de la ausencia de datos adecuados, para lo que empleó fuentes indirectas y entrevistas en profundidad. Durante los años ochenta y noventa se generalizaron las fuentes centradas en el uso del tiempo, lo que permitió la consolidación de la disciplina. El Consejo Superior de Investigaciones Científicas (CSIC), bajo la dirección de la profesora Durán, llevó a cabo encuestas periódicamente con la finalidad de conocer cómo se distribuyen las actividades en el interior de los hogares, y muy especialmente conocer y hacer visible el trabajo no remunerado (Durán y Rogero 2009). El Centro de Investigaciones sobre la Realidad Social (CIRES), el Instituto de la Mujer y el Instituto de Estadística del País Vasco (Eustat) han sido otras instituciones que han llevado a cabo encuestas centradas en el uso del tiempo.

No fue hasta los inicios del presente siglo cuando se llevó a cabo la primera encuesta propiamente denominada Encuesta de Empleo del Tiempo (2002-2003). Después se han realizado un buen número de trabajos en esta línea (González y Jurado 2009; Domínguez 2010; Sevilla-Sanz et al. 2010; Gutiérrez Domènech 2007; Ajenjo y García 2011; Domínguez 2012). La mayoría de trabajos tratan de las diferentes perspectivas a partir de las cuales se intentan explicar las desigualdades entre hombres y mujeres respecto a la división del trabajo no remunerado: i) disponibilidad de tiempo, ii) oportunidades relativas y iii) de género (Coltrane 2000). 
Desde la perspectiva de la disponibilidad de tiempo, el trabajo no remunerado que realiza cada miembro de la pareja está relacionado con el tiempo dedicado a trabajo remunerado (South y Spitze 1994). Así, en las parejas de doble ingreso, ambos miembros disponen de un tiempo similar si descontamos el que emplean en trabajo remunerado, de modo que es donde la división del trabajo no remunerado deviene más igualitaria (Ajenjo y García 2011; González y Jurado 2009). En esta misma línea, la mayor implicación en el trabajo no remunerado del hogar, por parte de los hombres, suele ser fruto de una reducción o concentración de su jornada laboral. En cuanto a las mujeres, su mayor participación en el trabajo remunerado se observa cuando liberan parte del trabajo familiar, ya sea externalizándolo, con una mayor implicación de otros miembros, o disminuyéndolo, tal y como ocurre con la reducción de la fecundidad (González y Jurado 2009). Además, el comportamiento difiere considerablemente los días laborales de los fines de semana, puesto que algunas tareas suelen aplazarse para los festivos (Sevilla-Sanz et al. 2010) siendo, además, los días en que los hombres realizan más trabajo doméstico (González y Jurado 2009).

Desde la perspectiva de las oportunidades relativas, el reparto del trabajo no remunerado está condicionado por el coste de oportunidad de cada miembro del hogar (Coltrane 2000; Bernhardt et al. 2008). Así, el fuerte incremento del nivel de estudios de la mujer comporta un incremento del coste de su dedicación a tareas domésticas, pero también un incremento de su poder de negociación. Ambas características comportan una mayor igualdad en el reparto del trabajo no remunerado en aquellas parejas donde la mujer tiene un elevado nivel de estudios (Blau 1998; Ajenjo y García 2011; González y Jurado 2009; Gutiérrez Domènech 2010; Domínguez 2012). Además, una mejor educación está correlacionada con unos mayores ingresos que, además de permitir la externalización de algunas tareas (González y Jurado 2009), mejora las condiciones de negociación. De modo que la cantidad de trabajo doméstico que realiza la mujer decrece con el nivel de ingresos (Sevilla-Sanz et al. 2010). Sin embargo, esta variación no es constante para todos los niveles de ingresos sino que encuentra su techo en el punto en que los ingresos de hombre y mujer se igualan, punto a partir del cual las diferencias en el tiempo dedicado a trabajo no remunerado se mantienen constantes (Sevilla-Sanz et al. 2010).

Esta circunstancia confirmaría la tercera de las perspectivas, denominada de género, $y$, según la cual, existen unas normas sociales bajo las cuales, a partir de un cierto momento, el efecto de igualación del trabajo doméstico desaparece (Coltrane 2000). Estas normas que rigen masculinidad y feminidad están mucho más arraigadas en la sociedad española que en otras (Sevilla-Sanz et al. 2010). Por otro lado, el efecto no es el mismo cuando se analiza por un lado el trabajo doméstico y por el otro el trabajo de cuidado de los hijos, puesto que únicamente se cumple en el primer caso. Este resultado lleva a la conclusión de que el trabajo doméstico estaría dominado por las normas de la masculinidad, bajo las cuales el hombre ha de eludir la realización de dicha actividad puesto que perjudica su estatus, mientras que el cuidado de los hijos se regiría por las normas de la feminidad, que reforzarían la voluntad de la mujer para responsabilizarse del cuidado de los hijos en primera instancia (Sevilla-Sanz et al. 2010). 
También los valores ideológicos explican parte de las diferencias en el reparto del tiempo entre hombre y mujer, de modo que unos valores más igualitarios reflejarán un reparto más igualitario del tiempo (Meil 2005). En este sentido, las parejas cohabitantes, fruto de los valores más igualitarios en que se suelen forjar, presentan un reparto más igualitario del tiempo (Baxter 2005; Batalaova y Cohen 2002; Domínguez y Castro 2008).

La edad y el ciclo de vida son otros elementos que se han considerado para explicar las desigualdades (Gershuny et al. 2005). El nacimiento de los hijos, por ejemplo, supone una reducción del tiempo productivo y un incremento del reproductivo por parte de las mujeres, mientras que apenas afecta a los varones, con el consiguiente incremento de la desigualdad (Gjerdingen y Center 2005; Dribe y Stanfors 2009; Domínguez 2012). En lo que se refiere al efecto de la edad, si bien es cierto que en las parejas más jóvenes se aprecia un reparto más igualitario de las tareas domésticas que induce a pronosticar una mayor igualdad en las nuevas generaciones (Gershuny et al. 1994; Robinson y Godbey 1999; Sayer 2005; Maclnnes 2005; Domínguez 2010), no queda claro el alcance real de este efecto y que no esté condicionado por las características intrínsecas de estas parejas, como que la cohabitación esté más extendida, tengan un nivel educativo más elevado y, sobre todo, por el hecho de no tener hijos (Ajenjo y García 2011).

Existe un consenso más o menos generalizado relativo a las características de la mujer, como su nivel de estudios o de ingresos, que son mucho más importantes que las de su pareja, llegándose incluso a la conclusión de que la participación de los hombres depende más de las características de su cónyuge que no de las propias (González y Jurado 2009). Aun así, algunos autores han analizado la existencia de un capital social generado por la mejor posición social del hombre y que permitiría a la mujer disponer de información para acceder a una mejor situación en el mercado remunerado y una división más igualitaria de las tareas domésticas (Bernardi 1999; Balcells 2009).

\section{OBJetivos E HIPÓTESIS}

Este trabajo tiene dos objetivos principales. El primero es analizar cómo han variado, si es que lo han hecho, las características y el uso del tiempo de la población en edad activa que vive en pareja entre los dos momentos de las encuestas. El segundo objetivo es analizar si, entre los dos momentos, las parejas han tendido a ser más igualitarias o, por el contrario, se siguen manteniendo las desigualdades de género en el uso del tiempo.

Para abordar el primer objetivo se ha tenido en cuenta que en el período de estudio se han dado importantes cambios en el mercado de trabajo. La reducción de la ocupación que se ha producido entre los dos momentos hace prever un descenso del tiempo dedicado a trabajo remunerado que se habrá consignado a otras actividades. Además, la desocupación ha afectado de manera desigual a hombres y mujeres con lo que también se prevé que los cambios difieran entre los dos sexos, pudiendo incidir en las desigualdades de género en el uso del tiempo. 
Respecto al segundo objetivo la principal hipótesis a contrastar es que, además de los cambios en las características de las parejas que favorecerían una mayor equidad entre hombres y mujeres, existe una evolución en la sociedad hacia una reducción de las desigualdades de género. Así pues, la hipótesis con la que se trabajará es si controlado por los diferentes factores que benefician una mayor igualdad entre hombres y mujeres hay una parte de las desigualdades atribuibles al momento de la encuesta. En caso de cumplirse esta hipótesis se podría considerar un signo de que la sociedad ha ganado en igualdad entre los dos períodos de estudio.

\section{FUENTES Y METODOLOGÍA}

Los datos utilizados pertenecen a la Encuesta de Empleo del Tiempo que el Instituto Nacional de Estadística llevó a cabo en los años 2002-2003 y 2009-2010. En ambos casos se recogen, para todos los miembros del hogar mayores de 10 años, todas las actividades realizadas durante 24 horas en intervalos de 10 minutos; además de una serie de variables sociodemográficas y económicas tanto a nivel de hogar como individual.

En la primera edición de la encuesta (2002-2003) se entrevistaron a 46.774 personas residentes en 20.603 hogares (Instituto Nacional de Estadística 2004). A partir de las relaciones de parentesco se han detectado 12.756 parejas, que una vez eliminadas aquellas en que alguno de sus miembros es menor de 15 años o mayor de 65 y aquellas en que falta información de algún miembro, han quedado reducidas a 9.487 parejas. En la segunda edición (2009-2010) se entrevistaron a 25.895 individuos pertenecientes a 9.541 hogares (Instituto Nacional de Estadística 2011). En este caso se han detectado 6.691 parejas, de las cuales 4.182 han formado parte de la muestra definitiva.

A pesar de que ambas encuestas utilizan cuestionarios similares, ha sido necesaria cierta homogeneización de las actividades y de las variables sociodemográficas más relevantes. Se ha analizado el tiempo empleado en un total de nueve actividades distintas:

1. Tiempo personal

2. Trabajo remunerado

3. Formación y otras actividades relacionadas

4. Tareas rutinarias del hogar (incluye cocinar, planchar, limpieza del hogar y de ropa...)

5. Tareas no rutinarias del hogar (incluye las reparaciones en el hogar, jardinería, cuidado de mascotas, compras, gestiones...)

6. Cuidado de niños y adultos

7. Ocio, deportes y aficiones

8. Desplazamientos

9. Otras actividades no específicas. 
Por otro lado, las variables sociodemográficas utilizadas han sido aquellas que según la literatura inciden en una distribución más igualitaria en el uso del tiempo entre los miembros de las parejas: el tipo de pareja, el tipo de unión', el nivel de estudios de la mujer, la disponibilidad de servicio doméstico, la presencia de hijos menores en el hogar y la edad de la mujer. Por otro lado, se han descartado algunas variables difíciles de armonizar entre ambas ediciones, otras con un alto nivel de no respuesta, así como otras por su escasa aportación al modelo.

Los resultados se dividen en tres apartados. En el primero se describe la evolución habida entre 2002-2003 y 2009-2010 en las características de las parejas. En el segundo se aborda el primer objetivo propuesto, analizando los cambios en el uso del tiempo, tanto a partir del porcentaje de población que realiza cada actividad, como el tiempo medio que dedican a la actividad aquellos que la realizan; haciendo especial hincapié en las diferencias entre ambos miembros de la pareja.

En el tercer apartado se trata el segundo objetivo, contrastar cómo ha evolucionado la diferencia entre hombres y mujeres en el uso del tiempo y, en concreto, en las cuatro actividades que presentan mayores cambios entre 2002-2003 y 2009-2010 -trabajo remunerado, tareas rutinarias del hogar, cuidado de niños y adultos, y ocio-, mientras que en el resto de actividades las diferencias apenas son perceptibles.

Para ello se ha utilizado el análisis multivariante de la varianza, tomando como variables dependientes la diferencia en el tiempo dedicado a cada una de las cuatro actividades entre el hombre y la mujer de cada pareja. En estos modelos se han fusionado los datos de ambas encuestas, creándose una variable que identifica el año al que pertenece la observación, variable que servirá para contrastar la principal hipótesis de trabajo. Así, el efecto del período de la encuesta mostrará si se tiende a una igualdad en el uso de tiempo o si las desigualdades de género se han mantenido o aumentado. Un resultado que será complementado, con el fin de evaluar la evolución de las diferencias hombre/mujer si las características de las parejas no se hubiesen modificado, con la incorporación sucesiva de variables, algunas de las cuales se han visto afectadas directamente por el cambio de ciclo económico. La edición de la encuesta, la disponibilidad de servicio doméstico y el día de la semana aparecen como variables de control en todos los modelos. En el segundo, además, se ha incorporado el tipo de pareja según la ocupación de ambos miembros. Para finalizar se ha elaborado un modelo completo, el cual introduce también el tipo de unión, el número de hijos, la edad de la mujer y su nivel de estudios.

${ }^{1}$ En la Encuesta 2009-2010 las parejas de hecho registradas se han clasificado en la misma categoría que las casadas. Esto puede dar una subestimación de la cohabitación en este período. 


\section{Características de las parejas}

El incremento de tipologías en las que la mujer se encuentra ocupada son indicadores de la evolución de la participación de la mujer en el trabajo remunerado (ver Tabla 1). Así, entre 2002-2003 y 2009-2010 se ha producido un incremento de parejas de doble ingreso que han pasado del $40,5 \%$ al $46,7 \%^{2}$, un incremento que se ha visto mermado por la recesión iniciada en los años anteriores a la segunda encuesta; como lo muestran los datos de la Encuesta de Población Activa del primer trimestre de 2008, donde la proporción de parejas de doble ingreso superaba el $50 \%$. Tal y como refleja la pirámide de población (ver Figura 1), la estructura por edad y sexo de estas parejas ha cambiado ligeramente, dándose un ligero envejecimiento tanto de los hombres como de las mujeres, fruto de la mayor incidencia del paro entre los jóvenes: la media de edad ha pasado de 41,5 a 42,9 años para los hombres y de 39,2 a 40,7 años para las mujeres.

El mayor cambio entre ambos años es el descenso de catorce puntos de las parejas en que solo trabaja el hombre, apreciándose pocas diferencias en la composición por edad. En el lado opuesto, la proporción de parejas en que solo trabaja la mujer se ha duplicado, variando significativamente su composición por edad con un rejuvenecimiento de casi cinco años para ambos sexos. Así, y como producto de una mayor desocupación entre los hombres, han incrementado considerablemente las parejas jóvenes-adultas que se encuentran en esta situación.

Por otro lado, las parejas en que no trabaja ningún miembro también han aumentado ligeramente, unos 2,5 puntos porcentuales; un aumento fruto de cierto descenso en un primer momento, hasta 2008, y de un rápido aumento posterior debido al fuerte incremento del paro. En lo referente a la composición por edades, la evolución es similar a las parejas en las que solo trabaja la mujer, aunque ahora habría que atribuir el rejuvenecimiento a la incidencia de la desocupación general entre los jóvenes-adultos.

A pesar de que los cambios han sido menores en el resto de características de las parejas, la mayor parte de estos indican un incremento de las características que la bibliografía muestra como más propensas a la igualdad hombre-mujer. Cabe destacar, en primer lugar, que al contrario de lo que sucede con la distribución según el tipo de pareja en función de la ocupación, en el resto de las variables los cambios se deben en mayor medida a un relevo generacional y no a un efecto coyuntural. Así, destaca sobre todo el crecimiento de la proporción de mujeres con estudios universitarios, que supera claramente a los hombres, y la de estudios secundarios, en detrimento de los estudios de primaria o secundaria. La consecuencia de la evolución dispar entre hombres y mujeres es el incremento de las parejas hipógamas, que supera por primera vez a las parejas hipérgamas.

\footnotetext{
${ }^{2}$ Estos porcentajes son consistentes con los observados a partir de la Encuesta de Población Activa. Según nuestras estimaciones serían del $42,0 \%$ en el primer trimestre de 2003 y 46,3\% en el primer trimestre de 2010.
} 
Otros cambios de menores consecuencias que se han producido entre los dos momentos son el incremento de las parejas cohabitantes y el aumento de la presencia de menores de 10 años en el hogar, fruto del continuado ascenso de la fecundidad hasta 2008. A diferencia de lo que sucede con el resto de características, la evolución de esta última va en detrimento de la igualdad hombre-mujer en el empleo del tiempo, tal y como sucede con la evolución de la disponibilidad de servicio doméstico, cuyo descenso como consecuencia de la crisis supone un hándicap para la igualdad.

Tabla 1.

Características de las parejas en 2002-2003 y 2009-2010 (\%).

\begin{tabular}{|c|c|c|}
\hline & $2002-2003$ & $2009-2010$ \\
\hline \multicolumn{3}{|l|}{ Tipo de pareja } \\
\hline Pareja de doble ingreso & 40,5 & 46,2 \\
\hline Pareja tradicional & 43,0 & 29,2 \\
\hline Solo trabaja la mujer & 5,0 & 10,7 \\
\hline No trabajan & 11,5 & 14,0 \\
\hline \multicolumn{3}{|l|}{ Tipo de unión } \\
\hline Cohabitantes & 7,1 & 12,1 \\
\hline Casados & 92,9 & 87,9 \\
\hline \multicolumn{3}{|l|}{ Nivel de estudios } \\
\hline \multicolumn{3}{|l|}{ Hombre } \\
\hline Sin estudios & 21,6 & 24,3 \\
\hline Primaria o ESO & 35,6 & 23,6 \\
\hline Bachillerato o FP & 26,6 & 32,9 \\
\hline Universitario & 16,2 & 19,3 \\
\hline \multicolumn{3}{|l|}{ Mujer } \\
\hline Sin estudios & 23,3 & 22,7 \\
\hline Primaria o ESO & 36,5 & 24,0 \\
\hline Bachillerato o FP & 23,8 & 30,2 \\
\hline Universitario & 16,4 & 23,1 \\
\hline Hipérgamas & 23,3 & 19,7 \\
\hline Homógamas & 56,2 & 56,4 \\
\hline Hipógamas & 20,5 & 23,9 \\
\hline \multicolumn{3}{|l|}{ Número de hijos } \\
\hline 0 & 64,7 & 59,2 \\
\hline 1 & 23,9 & 26,2 \\
\hline $2+$ & 11,4 & 14,6 \\
\hline \multicolumn{3}{|l|}{ Disponibilidad de servicio doméstico } \\
\hline Sí & 10,6 & 8,3 \\
\hline No & 89,4 & 91,7 \\
\hline
\end{tabular}

Fuente: Elaboración propia a partir de las Encuestas de Empleo del Tiempo 2002-2003 y 2009-2010 (Instituto Nacional de Estadística). 


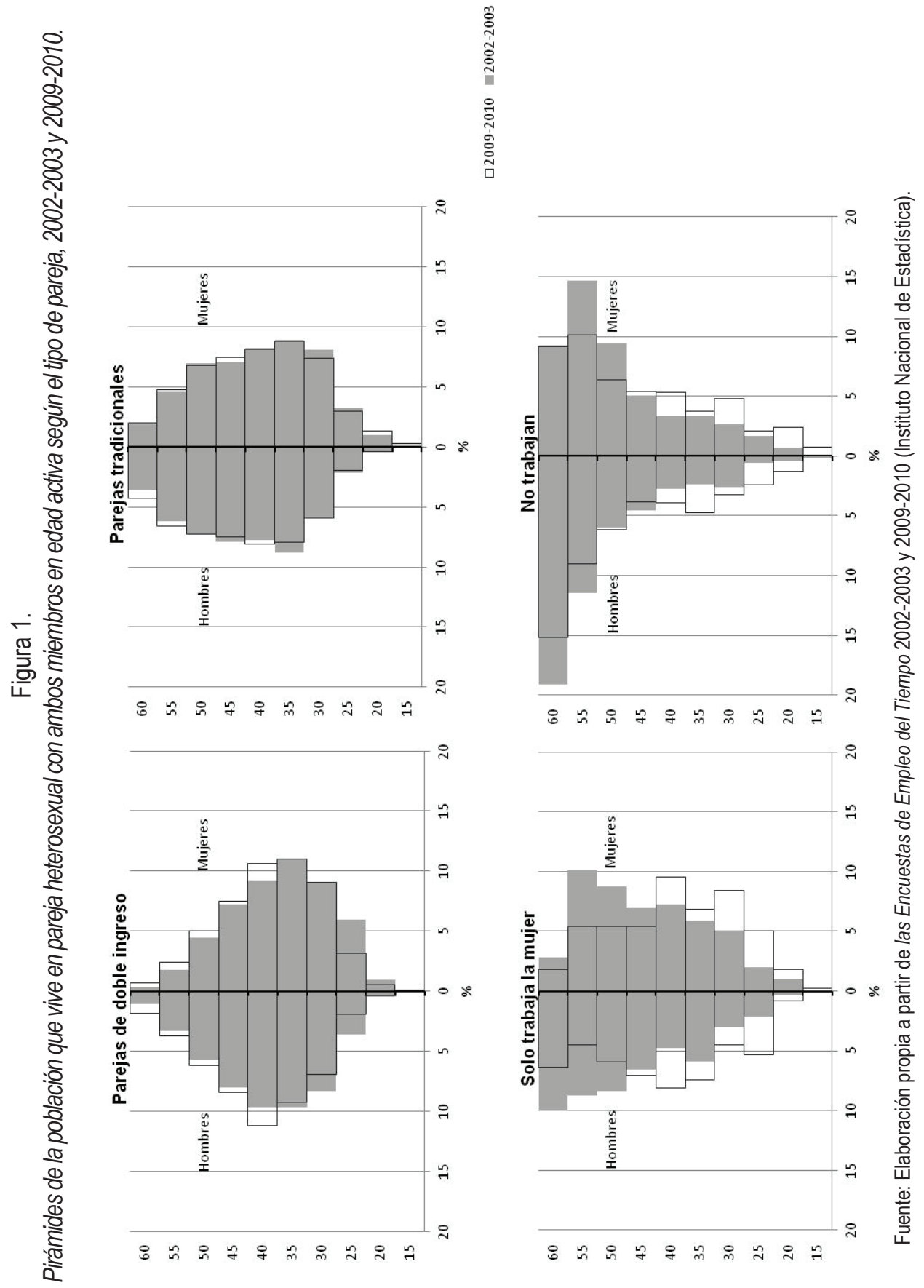




\section{Cambios en el uso del tiempo}

Una primera observación de las variaciones en el uso del tiempo (Tabla 2) muestra, en primer lugar, los efectos directos del incremento del paro masculino, que se traduce en un fuerte descenso del porcentaje de hombres que dedican al menos un minuto a trabajo remunerado, y, en segundo lugar, que entre las mujeres el incremento de la actividad ha sido superior al del paro, manifestándose en un mayor número de mujeres realizando trabajo remunerado. Evaluando el tiempo que dedican al trabajo remunerado, se observa también cierto cambio en las características de la ocupación, con un ligero aumento de las jornadas parciales que se traduce en un descenso del tiempo trabajado, más acusado entre los hombres que entre las mujeres (Tabla 3). Ambas evoluciones conducen a una disminución general de las diferencias entre hombres y mujeres, que aun así se mantienen, siendo los hombres los principales protagonistas de la esfera pública.

Respecto al trabajo doméstico rutinario, y a pesar de que ellas siguen siendo con creces las principales protagonistas, también se observa cierta tendencia a la igualdad, ya que, mientras los hombres han incrementado su participación, las mujeres la han disminuido muy levemente. Esta tendencia también se observa en el tiempo empleado en estas labores, donde cabe destacar que para el conjunto de la semana la diferencia entre mujeres y hombres ha pasado de tres horas diarias a dos, un descenso que se observa tanto los días laborables como los fines de semana.

Los menores cambios entre 2002-2003 y 2009-2010 se han producido en el conjunto de lo que hemos denominado las tareas no rutinarias del hogar. Estas labores, sobre todo cuando se llevan a cabo los días laborables, a pesar de que son más frecuentes entre las mujeres, son los hombres quienes al ejercerlas les dedican un mayor número de horas, algo que compensa el tiempo total. A pesar del equilibrio, cabe destacar que si analizamos separadamente tareas como las reparaciones, la jardinería o el cuidado de mascotas, de las compras y gestiones, las diferencias vuelven a manifestarse, siendo para ellos el primer grupo y para ellas el segundo.

En cuanto a la participación y al tiempo empleado en el cuidado de niños y adultos, se aprecia un incremento de participación generalizado, probablemente consecuencia de la mayor presencia de menores en los hogares por el incremento de la fecundidad, aunque ligeramente superior entre los hombres, lo que supone también cierto descenso de las diferencias.

En términos generales, ni la participación ni el tiempo dedicado al conjunto de actividades de ocio ha cambiado significativamente en estos años, empleando los hombres unos 45 minutos diarios más que las mujeres. Ahora bien, esta estabilización es producto de cambios importantes en la distribución de este ocio. Así, por ejemplo, incrementa considerablemente la participación tanto de hombres como de mujeres en aficiones y juegos, pero no el tiempo que le dedican, mientras que en la participación en actividades relacionadas con los medios de comunicación se observa un incremento mucho menor, 


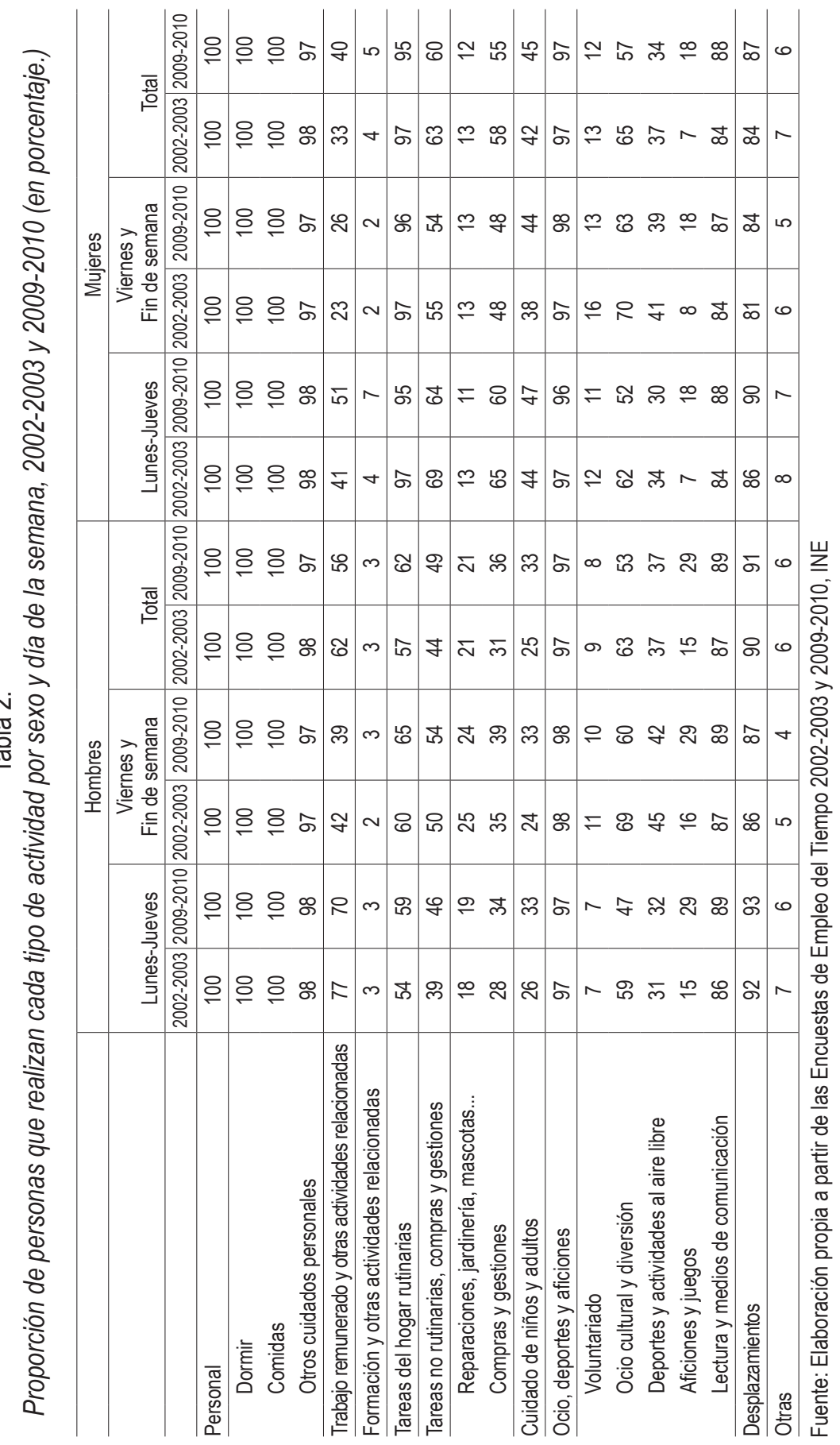




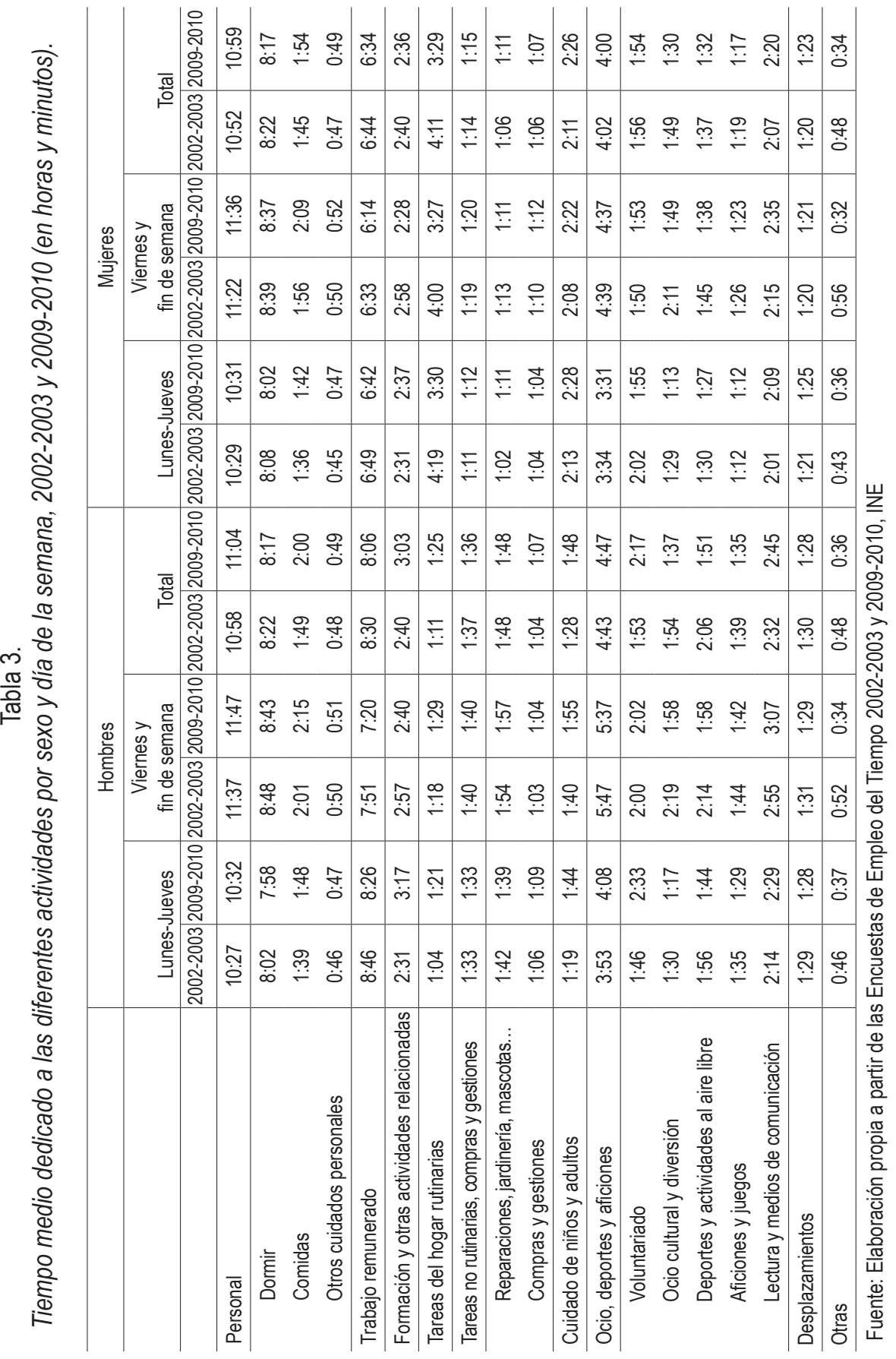


aunque aumenta considerablemente el tiempo empleado. Ahora bien, en el campo del ocio, el cambio más relevante se observa en el fuerte descenso tanto de la participación como del tiempo empleado en lo que se ha denominado como ocio cultural y diversión. Se trata, muy probablemente, de un efecto derivado de la crisis económica, la cual ha alterado los hábitos de ocio.

El resto de actividades presentan pocos cambios, tanto entre los dos períodos como entre sexos. Se trata de actividades básicas que realiza casi la totalidad de la población con lo que los márgenes de variación son bastante reducidos. Lo más destacable sería un ligero incremento de la movilidad de las mujeres, fruto posiblemente de la mayor participación en el mercado de trabajo, así como una dedicación un poco mayor a las comidas, de unos diez minutos de promedio, tanto entre hombres como entre mujeres.

\section{¿SON LAS PAREJAS MÁS IGUALITARIAS?}

La pregunta que aquí nos formulamos tiene que ver con la evolución de las diferencias en el uso del tiempo entre hombre y mujer en la primera década del siglo XXI. Tal y como nos muestran las dos encuestas del INE, la reducción de estas diferencias son evidentes, sobre todo en las tareas del hogar rutinarias y en el trabajo remunerado. Sin perder de vista que las mujeres siguen dedicando a trabajo doméstico mucho más tiempo que sus parejas y que los hombres emplean más tiempo en trabajo remunerado que sus cónyuges, entre 2002-2003 y 2009-2010 la diferencia entre ellos y ellas se han reducido considerablemente en ambos tipos de trabajo.

En la Tabla 4 se puede observar cómo han variado las diferencias entre el tiempo dedicado a las principales actividades por la mujer y el hombre según el tipo de pareja. Las diferencias en el tiempo dedicado a trabajo no remunerado rutinario son las que han experimentado un cambio más general ya que se ha producido en todos los tipos de pareja y para el conjunto de ellas ha disminuido en 1 hora. Por lo que respeta al trabajo remunerado las diferencias han decrecido también en una hora para el conjunto de parejas. Sin embargo, en este caso la variación no se ha producido en todas las tipologías, lo que denota que el cambio que se observa para el conjunto se debe más a cambios en la distribución de los tipos que a una reducción real de las diferencias.

Así pues, para analizar los cambios en la disminución de las desigualdades es necesario controlar por las características de las parejas, las cuales no son las mismas en los dos momentos, habiendo variado en el sentido en que se han incrementado aquellas más propensas a la igualdad. En los modelos MANOVA que se presentan a continuación se analiza el efecto de la evolución temporal controlando las características analizadas anteriormente, lo que nos permite analizar qué hubiera pasado si estas características no hubiesen variado.

Los modelos únicamente se presentan para las cuatro variables en las que se aprecian diferencias significativas entre hombres y mujeres. De este modo, no se muestran 
Tabla 4.

Diferencia entre la mujer y el hombre en tiempo dedicado a las principales actividades según la tipología de pareja por ocupación de sus miembros.

\begin{tabular}{|c|c|c|c|c|c|c|c|c|c|c|}
\hline & \multicolumn{2}{|c|}{$\begin{array}{c}\text { Pareja doble } \\
\text { ingreso }\end{array}$} & \multicolumn{2}{|c|}{$\begin{array}{c}\text { Pareja } \\
\text { tradicional }\end{array}$} & \multicolumn{2}{|c|}{$\begin{array}{c}\text { Sólo trabaja } \\
\text { la mujer }\end{array}$} & \multicolumn{2}{|c|}{$\begin{array}{c}\text { No trabaja } \\
\text { ninguno }\end{array}$} & \multicolumn{2}{|c|}{$\begin{array}{c}\text { Todas las } \\
\text { parejas }\end{array}$} \\
\hline & 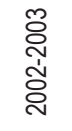 & $\begin{array}{l}\text { 을 } \\
\text { ஸे } \\
\text { ᄋे } \\
\text { ㅇ }\end{array}$ & 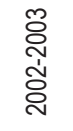 & $\begin{array}{l}\text { 울 } \\
\text { ஸे } \\
\text { oे }\end{array}$ & 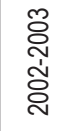 & $\begin{array}{l}\text { 을 } \\
\text { òे } \\
\text { ᄋे }\end{array}$ & 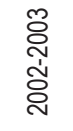 & $\begin{array}{l}\text { 을 } \\
\text { 웅 } \\
\text { ㅇ. }\end{array}$ & 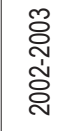 & $\begin{array}{l}\text { 을 } \\
\text { ஸे } \\
\text { ᄋे }\end{array}$ \\
\hline Trabajo no remunerado rutinario & 2:05 & $1: 40$ & $4: 35$ & $3: 52$ & $1: 28$ & $0: 39$ & $4: 23$ & $3: 24$ & $3: 23$ & $2: 27$ \\
\hline Trabajo no remunerado no rutinario & 0:01 & 0:00 & $0: 20$ & $0: 14$ & $-0: 43$ & $-0: 32$ & $-0: 26$ & $-0: 21$ & 0:04 & $0: 02$ \\
\hline Cuidado & $0: 19$ & $0: 25$ & $0: 54$ & 1:00 & $-0: 10$ & $-0: 09$ & $0: 16$ & $0: 17$ & $0: 32$ & $0: 30$ \\
\hline Trabajo remunerado & $-1: 30$ & $-1: 32$ & $-6: 02$ & $-5: 41$ & $4: 07$ & 4:02 & $-0: 15$ & $-0: 15$ & $-3: 01$ & $-1: 58$ \\
\hline Ocio & $-0: 47$ & $-0: 32$ & $0: 19$ & $0: 27$ & $-3: 25$ & $-3: 01$ & $-2: 50$ & $-2: 14$ & $-0: 41$ & $-0: 45$ \\
\hline Personal & $-0: 06$ & 0:02 & $0: 16$ & $0: 16$ & $-1: 08$ & $\mid-0: 46$ & $-0: 58$ & $-0: 45$ & $\mid-0: 06$ & $-0: 05$ \\
\hline
\end{tabular}

las diferencias en desplazamientos, cuidados personales y trabajo no remunerado no rutinario y compras. Además de mostrar pocas diferencias, los modelos para estas variables presentaban $r$ cuadrados muy pequeñas; un motivo más para excluirlos.

Para interpretar los coeficientes hay que tener en cuenta, en primer lugar, que las diferencias corresponden al tiempo dedicado por la mujer menos el tiempo dedicado por el hombre. En el caso del trabajo doméstico rutinario y de cuidados, por ejemplo, al ser más habitual que ella le dedique más tiempo que él, la media será positiva; de modo que cuando en una categoría el coeficiente sea negativo es sinónimo de mayor igualdad que la categoría de referencia. Por el contrario, al destinar los hombres más tiempo a trabajo remunerado y a ocio que las mujeres, los coeficientes negativos en una categoría comportan una mayor diferencia que la generada por la categoría de referencia.

La comparación de los coeficientes en los distintos modelos así como la evolución de la parte explicada ( $r$ cuadrado) permite matizar los resultados obtenidos y el efecto de cada variable. Así, la variabilidad explicada por el modelo es pequeña cuando únicamente se incorpora el momento de la encuesta, la disponibilidad de servicio doméstico y el día de la semana como variables de control, pero aumenta de forma muy importante al incorporar el tipo de pareja. Y aumenta sobre todo en las tareas del hogar rutinarias y en el trabajo remunerado, lo que pone de manifiesto la importancia de la ocupación para explicar las desigualdades de género es estos aspectos. La diferencia en el tiempo de cuidado queda mejor explicada al incorporar el resto de variables, en especial el número de hijos. 
Tabla 5.

MANOVA para la diferencia entre mujeres y hombres de tiempo dedicado a tareas del hogar rutinarias, cuidado de niños y adultos, trabajo remunerado y ocio. Modelo 1.

\begin{tabular}{|c|c|c|c|c|}
\hline & $\begin{array}{c}\text { Diferencia } \\
\text { trabajo } \\
\text { remunerado }\end{array}$ & $\begin{array}{c}\text { Diferencia } \\
\text { tareas hogar } \\
\text { rutinarias }\end{array}$ & $\begin{array}{c}\text { Diferencia } \\
\text { cuidado niños y } \\
\text { adultos }\end{array}$ & $\begin{array}{c}\text { Diferencia } \\
\text { tiempo de ocio }\end{array}$ \\
\hline \multicolumn{5}{|l|}{ Edición } \\
\hline $2002-2003$ & $-1: 06^{\star \star \star}$ & $0: 59^{* \star *}$ & 0:01 & 0:04 \\
\hline $2009-2010$ & ref & ref & ref & ref \\
\hline \multicolumn{5}{|c|}{ Disponibilidad de servicio doméstico } \\
\hline Sí & $1: 15^{\star \star \star}$ & $-1: 31^{\star \star \star}$ & $-0: 02$ & 0:04 \\
\hline No & ref & ref & ref & ref \\
\hline \multicolumn{5}{|l|}{ Día de la semana } \\
\hline Lunes-Jueves & $-1: 51^{* * *}$ & $0: 24^{* * *}$ & $0: 12^{* \star *}$ & $0: 39^{* * *}$ \\
\hline Viernes-Domingo & ref & ref & ref & ref \\
\hline Intersección & $-0: 59^{\star \star \star}$ & $2: 20^{\star \star *}$ & $0: 23^{* * *}$ & $-1: 08^{* * *}$ \\
\hline R2 & 0,051 & 0,058 & 0,005 & 0,012 \\
\hline
\end{tabular}

Fuente: Elaboración propia a partir de las Encuestas de Empleo del Tiempo 2002-2003 y 2009-2010, INE

Tabla 6.

MANOVA para la diferencia entre mujeres y hombres de tiempo dedicado a tareas del hogar rutinarias, cuidado de niños y adultos, trabajo remunerado y ocio. Modelo 2.

\begin{tabular}{|c|c|c|c|c|}
\hline & $\begin{array}{l}\text { Diferencia } \\
\text { trabajo } \\
\text { remunerado }\end{array}$ & $\begin{array}{l}\text { Diferencia } \\
\text { tareas hogar } \\
\text { rutinarias }\end{array}$ & $\begin{array}{c}\text { Diferencia } \\
\text { cuidado niños y } \\
\text { adultos }\end{array}$ & $\begin{array}{c}\text { Diferencia } \\
\text { tiempo de ocio }\end{array}$ \\
\hline \multicolumn{5}{|l|}{ Edición } \\
\hline $2002-2003$ & $-0: 05$ & $0: 38^{* * *}$ & $-0: 05^{\star *}$ & $-0: 17^{* * *}$ \\
\hline $2009-2010$ & ref & ref & ref & ref \\
\hline \multicolumn{5}{|l|}{ Tipo de pareja } \\
\hline Pareja tradicional & $-4: 26^{* * *}$ & $2: 18^{* * *}$ & $0: 35^{\star * *}$ & $1: 05^{* * *}$ \\
\hline Solo trabaja la mujer & $5: 33^{* * *}$ & $-0: 49^{* * *}$ & $-0: 31^{* \star \star}$ & $-2: 30^{\star * *}$ \\
\hline No trabajan & $1: 16^{* * *}$ & $2: 01^{* * *}$ & $-0: 04$ & $-1: 54^{* * *}$ \\
\hline Pareja de doble ingreso & ref & ref & ref & ref \\
\hline \multicolumn{5}{|c|}{ Disponibilidad de servicio doméstico } \\
\hline Sí & $0: 21^{* *}$ & $-0: 44^{* * *}$ & $0: 05^{*}$ & 0:06 \\
\hline No & ref & ref & ref & ref \\
\hline \multicolumn{5}{|l|}{ Día de la semana } \\
\hline Lunes-Jueves & $-1: 57^{\star \star \star}$ & $0: 27^{\star * *}$ & $0: 13^{* * *}$ & $0: 40^{* * *}$ \\
\hline Viernes-Domingo & ref & ref & ref & ref \\
\hline Intersección & $-0: 22^{* * *}$ & $1: 22^{* * *}$ & $0: 15^{\star * *}$ & $-0: 55^{\star * *}$ \\
\hline $\mathrm{R} 2$ & 0,396 & 0,237 & 0,056 & 0,163 \\
\hline
\end{tabular}

Fuente: Elaboración propia a partir de las Encuestas de Empleo del Tiempo 2002-2003 y 2009-2010, INE 
Tabla 7.

MANOVA para la diferencia entre mujeres y hombres de tiempo dedicado a tareas del hogar rutinarias, cuidado de niños y adultos, trabajo remunerado y ocio. Modelo completo

\begin{tabular}{|c|c|c|c|c|}
\hline & $\begin{array}{l}\text { Diferencia } \\
\text { trabajo } \\
\text { remunerado }\end{array}$ & $\begin{array}{c}\text { Diferencia } \\
\text { tareas hogar } \\
\text { rutinarias }\end{array}$ & $\begin{array}{c}\text { Diferencia } \\
\text { cuidado niños y } \\
\text { adultos }\end{array}$ & $\begin{array}{c}\text { Diferencia } \\
\text { tiempo } \\
\text { de ocio }\end{array}$ \\
\hline \multicolumn{5}{|l|}{ Edición } \\
\hline $2002-2003$ & $-0: 05$ & $0: 31^{* \star \star}$ & $-0: 00$ & $-0: 17^{\star \star \star}$ \\
\hline $2009-2010$ & ref & ref & ref & ref \\
\hline \multicolumn{5}{|l|}{ Tipo de pareja } \\
\hline Pareja tradicional & $-4: 17^{\star * \star}$ & $1: 57^{\star * *}$ & $0: 35^{\star \star *}$ & $1: 09^{* * *}$ \\
\hline Solo trabaja la mujer & $5: 35^{\star \star \star}$ & $-1: 08^{* \star *}$ & $-0: 20^{\star \star \star}$ & $-2: 29^{\star \star \star}$ \\
\hline No trabajan & $1: 24^{\star \star *}$ & $1: 13^{\star * *}$ & $0: 14^{\star \star \star}$ & $-1: 48^{\star \star \star}$ \\
\hline Pareja de doble ingreso & ref & ref & ref & ref \\
\hline \multicolumn{5}{|l|}{ Tipo de unión } \\
\hline Cohabitante & $-0: 00$ & $-0: 20^{* * *}$ & 0:01 & $-0: 01$ \\
\hline Casados & ref & ref & ref & ref \\
\hline \multicolumn{5}{|l|}{ Nivel de estudios de la mujer } \\
\hline $\begin{array}{l}\text { Sin estudios o primaria } \\
\text { incompleta }\end{array}$ & $-0: 27^{\star * *}$ & $1: 12^{\star * *}$ & $-0: 05^{*}$ & $-0: 19^{\star * *}$ \\
\hline Primaria o ESO & $-0: 26^{\star * *}$ & $1: 00^{\star * *}$ & $-0: 00$ & $-0: 11^{\star *}$ \\
\hline Bachillerato o FP & $-0: 21^{\star \star *}$ & $0: 31^{* * *}$ & $0: 02$ & $-0: 10^{*}$ \\
\hline Universitario & ref & ref & ref & ref \\
\hline \multicolumn{5}{|l|}{ Número de hijos } \\
\hline Sin hijos & $0: 37^{\star \star \star}$ & $0: 06$ & $-1: 21^{\star \star *}$ & $0: 22^{* * *}$ \\
\hline 1 & 0:05 & $0: 03$ & $-0: 20^{\star * *}$ & 0:05 \\
\hline $2+$ & ref & ref & ref & ref \\
\hline \multicolumn{5}{|l|}{ Edad } \\
\hline$<35$ & $0: 26^{* * *}$ & $-1: 17^{* * *}$ & $0: 11^{* * *}$ & $0: 15^{* * *}$ \\
\hline $35-49$ & $0: 10$ & $-0: 27^{\star * \star}$ & $0: 02$ & $0: 04$ \\
\hline $50+$ & & & & ref \\
\hline \multicolumn{5}{|c|}{ Disponibilidad de servicio doméstico } \\
\hline Sí & $0: 19^{* *}$ & $-0: 29^{* * *}$ & $-0: 03$ & $0: 05$ \\
\hline No & ref & ref & ref & ref \\
\hline \multicolumn{5}{|l|}{ Día de la semana } \\
\hline Lunes-Jueves & $-1: 57^{\star * \star}$ & $0: 28^{* \star *}$ & $0: 13^{\star \star *}$ & $0: 40^{* * *}$ \\
\hline Viernes-Domingo & ref & ref & ref & ref \\
\hline Intersección & $-0: 42^{* * *}$ & $1: 26^{* * *}$ & $1: 02^{* * *}$ & $-1: 08^{\star \star *}$ \\
\hline R2 & 0,398 & 0,300 & 0,205 & 0,165 \\
\hline
\end{tabular}

Fuente: Elaboración propia a partir de las Encuestas de Empleo del Tiempo 2002-2003 y 2009-2010, INE. 
Haciendo un primer repaso a las 4 variables dependientes en conjunto, y salvo algunas excepciones que comentaremos más adelante, existen dos factores claramente favorables a la disminución de las desigualdades: la ocupación y la educación femenina. También se intuye una menor desigualdad en fin de semana, excepto en el tiempo de ocio. A continuación, veamos para cada variable los cambios más significativos.

\section{Trabajo remunerado}

La fuerte reducción de las diferencias entre hombres y mujeres, en cuanto al tiempo destinado a trabajo remunerado debe atribuirse, exclusivamente, al cambio de las características laborales de las parejas, es decir, al fuerte incremento de las parejas donde solo trabaja la mujer y de las parejas de doble ingreso: a igualdad de tipo de pareja, el cambio entre 2002-2003 y 2009-2010 deviene no significativo. La diferencia es ligeramente superior a la hora en el primer modelo, pero prácticamente desaparece al incorporar la tipología de pareja.

Así, pues, el cambio debe atribuirse, en primer lugar, al fuerte incremento de la actividad femenina y, en consecuencia, de su ocupación; y, en segundo lugar, a cierto descenso de la ocupación masculina. Ambas características conllevan evidentemente a la reducción de las diferencias en actividad.

Destacamos, del resto de variables, que las parejas sin hijos tienen un reparto más igualitario, consecuencia de que suelen ser las madres las que reducen su jornada laboral para atender a los hijos.

\section{Tareas del hogar rutinarias}

Las diferencias entre hombres y mujeres en cuanto al tiempo empleado en tareas domésticas rutinarias han descendido considerablemente en menos de una década. Ahora bien, ¿esta reducción es solamente consecuencia de cambios en la actividad, o se habría producido también si la actividad no hubiese cambiado?

Según el primer modelo generado (Tabla 5), la reducción atribuible al cambio temporal es de una hora. Gran parte de esta diferencia debe atribuirse a los cambios en la ocupación, de modo que se reduce a 38 minutos cuando incorporamos el tipo de pareja (Tabla 6). A pesar de ello, y al contrario de lo que sucede con el trabajo remunerado, los datos muestran que, aunque no hubiera habido cambios en la ocupación, la diferencia en el tiempo que mujeres y hombres destinan a tareas del hogar rutinarias hubiese descendido, y lo hubiese hecho en unos cuarenta minutos diarios. Esta conclusión muestra que la tendencia hacia un reparto más igualitario de las tareas domésticas está muy consolidada, y que posiblemente proseguirá en los próximos años en los dos sentidos en que ha venido haciéndolo hasta ahora: por la reducción de las diferencias de actividad entre hombres y mujeres, y por una mayor igualdad en el seno de las parejas.

Aventuramos esta primera conclusión porque, incluso controlando algunas de las características de las parejas que la literatura muestra como importantes, las diferencias 
entre los dos momentos siguen siendo significativas (Tabla 6). Nos referimos, sobre todo, al nivel de estudios de la mujer y a la cohabitación. La tendencia en ambas ha significado un incremento relativo entre 2002-2003 y 2009-2010, incremento que juega a favor de la igualdad en los tiempos que hombres y mujeres destinan a trabajo no remunerado, ya que las diferencias suelen ser menores en las parejas cohabitantes y en aquellas en que la mujer dispone de un mayor nivel de estudios (Blau 1998; Batalova y Cohen 2002; Baxter 2005; González y Jurado 2009; Domínguez 2012). Pues bien, la reducción de la diferencia entre hombres y mujeres en tareas del hogar rutinarias seguiría siendo significativa (de media hora aproximadamente), incluso si estas características no hubiesen cambiado, conclusión que reafirma la tendencia hacia una mayor igualdad.

\section{Trabajo de cuidado}

La diferencia entre hombre y mujeres en cuanto al tiempo dedicado al cuidado, ya sea de niños 0 adultos, prácticamente no ha cambiado en estos años. Las mujeres siguen dedicando al cuidado más tiempo que los hombres, una diferencia que aumenta los días laborables. Ahora bien, cuando al modelo se introduce el tipo de pareja, el cambio entre 2002-2003 y 2009-2010 pasa a ser significativo y además en sentido inverso al esperado, de modo que la diferencia entre hombres y mujeres, aunque no de forma importante, ha aumentado estos años.

El aumento de la fecundidad, y el consiguiente aumento de la presencia de niños a cargo, se encuentra detrás de este incremento de la diferencia. Y es que solamente con el hecho de introducir la variable número de hijos en el hogar, el cambio entre 2002-2003 y 2009-2010 vuelve a no ser significativo.

Resulta también interesante destacar que en este caso el efecto de la educación de la mujer es prácticamente nulo e incluso el único valor significativo corresponde a las mujeres menos educadas, entre las cuales la diferencia se reduce.

\section{Ocio}

En cuanto al tiempo empleado en ocio, y en concordancia con los resultados descriptivos, las diferencias entre hombres y mujeres apenas han cambiado entre los dos momentos (Tabla 5). Los hombres destinan más tiempo a ocio que las mujeres, sobre todo los fines de semana, reduciéndose la diferencia entre semana unos 40 minutos. Ahora bien, a igualdad del resto de variables sí que parece que las diferencias han descendido en unos 20 minutos (Tabla 7), una reducción que ya se percibe al introducir la variable tipo de pareja (Tabla 6).

También se refleja en el modelo el efecto negativo que tiene la presencia de los hijos en las desigualdades en el tiempo de ocio. En las parejas sin hijos la desigualdad es unos 20 minutos menor que en las parejas con algún hijo.

El efecto del tipo de pareja es muy particular si lo comparamos con los coeficientes observados para el resto de variables dependientes, y es que es en las parejas tradi- 
cionales donde se estima una mayor igualdad en tiempo dedicado a ocio: las parejas en que la mujer no trabaja ven reducidas su desigualdad en tiempo de ocio en una hora respecto a aquellas en las que trabajan ambos. En el lado opuesto, para las parejas en que solo ellas trabajan, la diferencia crece en dos horas y media a favor del hombre respecto a las parejas de doble ingreso. Este dato parece lógico si tenemos en cuenta que ellas están inmersas en el mercado laboral y ellos no. Sin embargo, no es tan evidente que las desigualdades también se amplíen a favor del hombre para las parejas en que ambos no trabajan y, menos aún, que aumente en casi dos horas respecto a las parejas de doble ingreso.

\section{CONCLUSIONES}

El reparto del tiempo en las parejas ha cambiado entre los dos períodos estudiados. Aunque parte de los cambios reflejan las tendencias que se han dado y se están dando en otros países y que en gran medida son consecuencia de los cambios en las características de las parejas, no debemos olvidar que la coyuntura económica es también un factor importante a la hora de analizar los cambios acontecidos.

Si bien la tendencia en los últimos años había sido un incremento de la ocupación femenina y una estabilización de la masculina, la coyuntura ha comportado un descenso en ambas, aunque mayor en la masculina, de modo que las desigualdades han seguido reduciéndose. Paralelamente, otro cambio en el trabajo remunerado atribuible a la coyuntura ha sido el descenso del tiempo empleado por parte de ambos.

Respecto del trabajo doméstico rutinario, la coyuntura ha acentuado la tendencia a una mayor igualdad que ya se estaba produciendo. En este sentido cabe destacar, en primer lugar, que el tiempo total dedicado al hogar se ha reducido los últimos años, y que esta reducción global, acompañada de cierto incremento por parte de los hombres, ha comportado un descenso considerable del tiempo que las mujeres emplean en tareas del hogar. En cambio, en cuanto a las actividades de cuidado a niños y adultos, ambos sexos aumentan su tiempo y, a pesar de que el incremento es mayor en ellos que en ellas, las mujeres siguen dedicando un tiempo muy superior.

En relación al tiempo de ocio, se dedica más tiempo a la lectura y a los medios de comunicación y menos a actividades como cine, teatro, etc. Este descenso hace suponer un cambio en los hábitos de ocio, fruto de la crisis, que se traduciría en una reducción de las actividades de un coste mayor y un incremento de aquellas presumiblemente más económicas. En las actividades de ocio las desigualdades son mayores en los fines de semana, al contrario que el resto de actividades que aumentan las diferencias en días laborables.

Las características de las parejas también han cambiado en la última década y lo han hecho principalmente incrementándose aquellas parejas de características más propensas a un reparto de tiempo más igualitario. Han aumentado las parejas de doble ingreso 
y, fruto de la incidencia desigual de la desocupación, también las parejas en que solo trabaja la mujer. Del mismo modo, crece la proporción de mujeres con educación superior, y como consecuencia, cada vez es mayor el número de parejas en que ellas tienen una educación superior a su cónyuge. También sigue extendiéndose la cohabitación que se suele asociar a valores más igualitarios y a una menor especialización de los roles. En contrapeso, algunas características que suelen ir acompañadas de una mayor desigualdad también se han incrementado, como es la presencia de hijos menores en el hogar.

Pero, aparte de los cambios en las parejas que habrían producido de por sí una disminución de las desigualdades, nos preguntamos si hay una reducción de la desigualdad debida a otros factores distintos a los cambios en las características de las parejas; es decir, si el cambio se hubiese producido sin que las características hubiesen cambiado y, en caso afirmativo, medir la magnitud de este cambio.

Y la respuesta es claramente afirmativa. Los resultados muestran que las desigualdades se habrían reducido aun habiéndose mantenido las características de las parejas. Y lo habrían hecho en dos de los ámbitos donde mayores diferencias se aprecian en el tiempo que dedican hombres y mujeres, como son las tareas domésticas rutinarias y el tiempo de ocio. El valor de la reducción no es excesivo pero hay que tener en cuenta que se ha producido en un espacio de tiempo relativamente corto. En términos absolutos, la reducción de las diferencias en trabajo doméstico ha sido de más de una hora; y, controlando por el resto de variables, de 31 minutos. En el tiempo de ocio la reducción se estimaría en unos 17 minutos. Así pues, creemos que hay que valorar positivamente esta reducción y considerar que aunque sea a un ritmo lento las desigualdades se están reduciendo: por una parte porque las características de las parejas son más igualitarias, destacando una mayor ocupación y un mayor nivel educativo de las mujeres; y, por otra, porque se está produciendo un cambio hacia una mayor equidad, al menos en lo que al reparto del tiempo se refiere.

Así, creemos que el componente estructural del cambio existe y que la ganancia de poder de la mujer le está permitiendo reducir las desigualdades en la vida privada.

\section{RefERENCIAS BibLIOgRáfICAS}

Aguiar, M., E. Hurst y L. Karabarbounis. 2011. "Time use during recession". NBER Working Paper 17259, National Bureau of Economic Research, Cambridge.

Ajenjo, M. y J. García-Román. 2011. "El tiempo productivo, reproductivo y de ocio en las parejas de doble ingreso". Papers. Revista de Sociología 96:985-1006.

Balcells, L. 2009. "Analyzing the division of household labor within Spanish families". Revista Internacional de Sociología 67:83-105.

Batalova, J. A. y P. N. Cohen. 2002. "Pre-marital cohabitation and housework: couples in cross national perspective". Journal of Marriage and Family 64:743-755.

Baxter, J. 2005. "To marry or not to marry: marital status and the household division of household". Journal of Marriage Issues 26:300-321. 
Becker, G. 1985. "Human capital, effort, and sexual division of labor". Journal of Labor Economic 3:33-58.

Becker, P. E. y P. Moen. 1999. "Scaling back: dual-earner couples' work family strategies". Journal of Marriage and the Family 61:995-1007.

Berk, S. F. 1985. The gender factory: The apportionment of work in American households. Nueva York: Plenum.

Bernardi, F. 1999. "Does the husband matter? Married women and employment in Italy". European Sociological Review 15:285-300.

Bernhardt, E., T. Noack y T. H. Lyngstad. 2008. "Shared Housework in Norway and Sweden: advancing the gender revolution". Journal of European Social Policy 18:275-288.

Bianchi, S. M., J. P. Robinson y M. Milkie. 2006: Changing rhythms of American family life. Nueva York: Russell Sage

Bittman, M. y J. Wajcman. 2000. "The Rush Hour: The Character of Leisure Time and Gender Equity". Social Forces 79:165-189.

Blau, F. 1998. "Trends in the well-being of American Women". Journal of Economic Literature 36:112-165.

Coltrane, S. 2000. "Research on Household Labor: Modeling and Measuring the Social Embeddedness of Routine Family Work". Journal of Marriage and Family 62:1208-1233.

Dema, S. 2005. "Entre la tradición y la modernidad: las parejas españolas de doble ingreso". Papers 77:135-155.

Domínguez, M. у T. Castro. 2008. "Women's changing socioeconomic position and union formation in Spain and Portugal". Demographic Research 19:1513-1550.

Domínguez, M. 2010. “¿Cada vez más igualitarios? Los valores de género de la juventud y su aplicación práctica". Revista de estudios de juventud 90:103-122.

Domínguez, M. 2012. "La división del trabajo doméstico en las parejas españolas. Un análisis de uso del tiempo". Revista Internacional de Sociología 70:153-179.

Dribe, M. y M. Stanfors. 2009. "Does Parenthood Strengthen a Traditional Household Division of Labor? Evidence from Sweden". Journal of Marriage and the Family 71:33-45.

Durán, M. Á. y J. Rogero-García. 2009. La investigación sobre el uso del tiempo. Madrid: CIS.

Ferree, M. M. 1991. "The Gender Division of Labor in Two-Earner Marriages: Dimensions of Variability and Change". Journal of Family Issues 12:158-180.

Gershuny, J., M. Godwin y S. Jones. 1994. "The domestic labour revolution: a process of lagged adaptation". Pp 151-197 en The social and Political Economy of the household, editado por M. Anderson et al. Oxford: Oxford University Press.

Gershuny, J. 2000. Changing times: work and leisure in post-industrial society. Oxford: Oxford University Press.

Gershuny, J., M. Bittman y J. Brice. 2005. "Exit, Voice, and Suffering: Do Couples Adapt to Changing Employment Patterns?". Journal of Marriage and Family 67:656-665.

Gjerdingen, D. K. y B. A. Center. 2005. "First-Time Parents and Postpartum Changes in Employment, Childcare and Housework Responsabilities". Social Science Research 34:103-116. 
González, M. J. y T. Jurado. 2009. “¿Cuándo se implican los hombres en las tareas domésticas? Un análisis de la Encuesta de Empleo del Tiempo". Panorama Social 10:65-81.

Gutiérrez-Domènech, M. 2010. "Parental Employment and Time with Children in Spain". Review of Economics of the Household 8:371-391.

Hochschild, A. R. 1989. The second shift: working parents and the revolution at home. New York: VikingPress.

Instituto Nacional de Estadística (INE). 2004. Encuesta de Empleo del Tiempo 2002-2003. Tomo I. Metodología y Resultados Nacionales. Consultado 05/10/2011 (http://www.ine.es/daco/daco42/ empleo/empleotiempo03_metynac.pdf).

Instituto Nacional de Estadística (INE). 2011. Encuesta de Empleo del Tiempo 2009-2010. Metodología. Consultado 05/10/2011 (http://www.ine.es/metodologia/t25/t25304471.pdf).

MacInnes, J. 2005. "Diez mitos sobre la conciliación de la vida laboral y familiar". Cuadernos de Relaciones Laborales 23:35-71.

Meil, G. 2005. "El reparto desigual del trabajo doméstico y sus efectos sobre la estabilidad de los proyectos conyugales". Revista Española de Investigaciones Sociológicas, 111:163-180.

Robinson, J. y G. Godbey. 1997. Time for Life-The Surprising Ways People Use Their Time. Penn States Press.

Sayer, L. 2005. "Gender, time and inequality: Trends in women's and men's paid work, unpaid work and free time". Social Forces 84:285-303.

Sevilla-Sanz, A., J. I. Giménez-Nadal y C. Fernández. 2010. "Gender roles and the division of unpaid work in Spanish Households". Feminist Economics 14:137-184.

South, S. J. y G. Spitze. 1994. "Housework in Marital and Nonmarital Households". American Sociological Review 59:327-347.

West, C. y Zimmermann, D. H. 1987. "Doing Gender". Gender\&Society 1:125-151.

Windebank, J. 2001. "Dual-earner couples in Britain and France: Gender divisions of domestic labour and arenting work in different welfare states". Work Employment Society 15:269-290.

Marc Ajenjo Cosp es Licenciado en Sociología y Doctor en Geografía Humana (Demografía) por la Universitat Autònoma de Barcelona. Imparte docencia como Investigador postdoctoral del Departamento de Sociología, siendo además investigador asociado al Centre d'Estudis Demogràfics. Ha sido investigador principal del proyecto I+D+I El reparto del tiempo y el uso del espacio en las parejas biactivas (Ref. CSO2009-08273), colaborando actualmente en el I+D+I Espacios de vida y usos del tiempo de las familias post divorcio (CSO2012-39157).

Joan García Roman es Doctor en Demografia por la Universitat Autonoma de Barcelona. Ha formado parte de distintos proyectos de investigacion vinculados al Centre d'Estudis Demografics entre ellos el proyecto WORLDFAM financiado por la European Research council ((ERC-2009StG-240978) y el proyecto I+D+I El reparto del tiempo y el uso del espacio en las parejas biactivas (Ref. CSO2009-08273). Actualmente trabaja como investigador asociado en el Minnesota Population Center de la University of Minnesota.

RECIBIDO: 28/05/2012

ACEPTADO: 28/03/2013

Publicado on-line: 12/03/2014

RIS, VOL.72. No 2, MAYO-AGOSTO, 453-476, 2014. ISSN: 0034-9712. DOI: 10.3989/ris.2012.05.28 